\title{
Multimass King models with Kroupa mass function
}

\author{
Marco Merafina*t \\ Department of Physics, University of Rome "La Sapienza", p.le A. Moro 5, I-00185 Rome (Italy) \\ E-mail: marce.merafinadromal.infn.it
}

\begin{abstract}
Multimass models for globular clusters are introduced by using Kroupa mass function. These models generalize the single mass King models presented last year in "XII Multifrequency Behaviour of High Energy Cosmic Sources Workshop" (2017), where the thermodynamical instability of such models was also discussed. Observational profiles of surface brightness of some clusters are compared and contrasted with the theoretical predictions in new and old models. Effects of mass segregation on the distribution of stars and the consequences on the equipartition of the energy among the stars are also considered, implying different conclusions about the thermodynamical instability.
\end{abstract}

Frontier Research in Astrophysics - III (FRAPWS2018)

28 May - 2 June 2018

Mondello (Palermo), Italy

\footnotetext{
* Speaker.

$\dagger$ in collaboration with INAF - Osservatorio Astronomico di Roma.
} 


\section{Introduction}

Globular clusters (GCs) are spherical stellar systems essentially without gas or recent star formation episodes, dust or younger elements. Their total mass can be found in the range $10^{4} M_{\odot} \div$ $10^{6} M_{\odot}$. Around the Milky Way it has been possible to survey a large number of these systems: Harris in 1996 catalogued more than 150 members [ 6$]$. These are privileged targets for observations since it is possible to separate their single star components [四].

Moreover, GCs are the best possible laboratories to test the effects of thermodynamics on their dynamical evolution, due to the collisions among stars, having a relatively small relaxation time with respect to their age ranging between 10 to 13 Gyr. They are probably the only "point of contact" between gravity and thermodynamics, and a complete analysis of this aspects allows the individuation of the onset of gravothermal instability inducing the core collapse, leading to conclude that this collapse occurs well before than commonly believed [14]].

Numerical simulations are also an important and powerful tool to check the validity of the theoretical models, but they enforce us to many limitations. Time of calculus is one of the most significant because simulating $\mathrm{N}$-body systems with $10^{5} \div 10^{6}$ stars requires several months of computing time if one have not a very powerful calculator [ㅁ] ]. We need to simplify our work by making assumptions which sometimes keep us away from studying all phenomena occuring in the reality and therefore it is necessary to think new ways of implementing cluster models to be able to take into account as many aspects as possible.

One important aspect of GCs is the spectrum of mass, which is often set aside in simulation and modeling by assuming that all the stars have a constant mass value. In most cases this assumption seems to be in accordance with observations, expecially in the central regions of the clusters [ $[\mathrm{B}]$ [龱], but it moves us away from a correct analysis of the outermost regions of these systems. In fact, stars with heavier masses tends to sink in the core, which is heavier than the single mass case, while the lighter components gain higher velocities and widen the outermost regions. This phenomenon is called mass segregation, which has implication in the evaporation of the stars and the core collapse, and affects the evolution and stability of these systems. Nowadays, observational evidences of this phenomenon stand without any question (see e.g. [B] [G][2] ).

\section{The multimass King model: Da Costa-Freeman}

The King distribution has been mostly applied ignoring the mass spectrum of the GCs. This assumption seems to work well for several clusters, since comparison between the surface density of the theoretical models and the luminosity profiles of the observed GCs in our galaxy shows a fine match. However, this is an approximation that does not allow a full understanding of many phenomena of these systems.

The mass spectrum modifies the luminosity profile of a globular cluster. Stars with greater masses affect the luminosity of the system, whereas the lighter elements, which dominate in number, mainly affect the gravitational potential [2]. There are few GCs which show a significant deviation of the luminosity profile from the theoretical single mass model. Da Costa \& Freeman pointed out how the GCs 47 Tuc, M3 and M15 show a luminosity profile divergence from the theoretical model. In the central regions of these systems we obtain a good fit, but not in the outermost 
regions. So that, when we compare numerical simulations with observed data, there are details we cannot see and understand if we do not look at the theory.

In star clusters with a mass spectrum, elements with different mass intervals can have different distributions in radius due to energy equipartition, which involves the kinetic energy of the different mass classes. Thus, for example, if the system tends to equipartition, heavier masses have lower velocities and cannot escape from the central regions with large values of the gravitational potential []ㅐ]. As the system tends to equipartition, these elements sink to the core and loose energy to the lighter stars, which can move to the external region and also evaporate.

Mass spectrum can also affect stability of these systems: if the ratio between relative density of higher mass stars and lower mass is sufficiently high, the low mass stars cannot achieve all the kinetic energy released by the greater ones and we have no more equilibrium and equipartition. In such a case, the heavy core of the globular cluster would collapse, with a disruption of the outer regions.

In 1976, Da Costa \& Freeman [ []] developed a dynamical model using the mass spectrum and the observed luminosity function, finding a better representation of the brightness profile of M3, starting from the King distribution function [ [ ] ] in terms of the energy. They assumed that the stars of the globular cluster can be grouped into discrete mass classes. Each of these has stars with mass $m_{i}$. The distribution in phase space was given by

$$
f_{i}(r, v)=\alpha_{i}\left[e^{E_{i} / m_{i} \sigma_{i}^{2}}-e^{C / \sigma_{i}^{2}}\right] \quad \text { with } \quad E_{i} \leq-m_{i} C,
$$

where $\sigma_{i}$ was related to the velocity dispersion of the stars of the i-mass class and $C>0$ was a constant connected to the normalization of the gravitational potential $\varphi\left(r_{t}\right)$ at the edge of the system corresponding to tidal radius $r_{t}$, while $\alpha_{i}$ was a normalizing number factor also related to the i-mass class. The energy for each mass class was given by

$$
E_{i}=\frac{1}{2} m_{i} v^{2}+m_{i} \varphi(r)
$$

The authors generated a model for a particular cluster varying the number factors $\alpha_{i}$ and the value of the central potential $\varphi_{0}$, iteratively, until making the model consistent with the data. They verified that the virial theorem was satisfied for all the models to better than $0.01 \%$ of the dimensionless kinetic energy, and that the models were indistinguishable from those of King [ [8]. Finally they compare the theoretical model to the observative data by fitting the surface brightness profile for M3 (NGC 5272). The fit is shown in Fig.Шl.

\section{Multimass model with continuous mass function}

Da Costa \& Freeman [వ] generated the multimass model by dividing the mass spectrum in mass classes and using the average value for each of them. They found a good agreement with the observational data in M3. There are several papers all considering multimass models with a

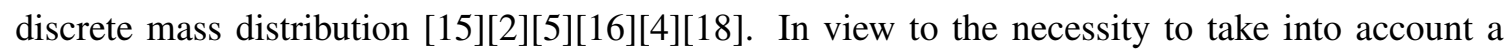
continuous mass function for the distribution of stars in a globular cluster, we consider the Kroupa mass function. 


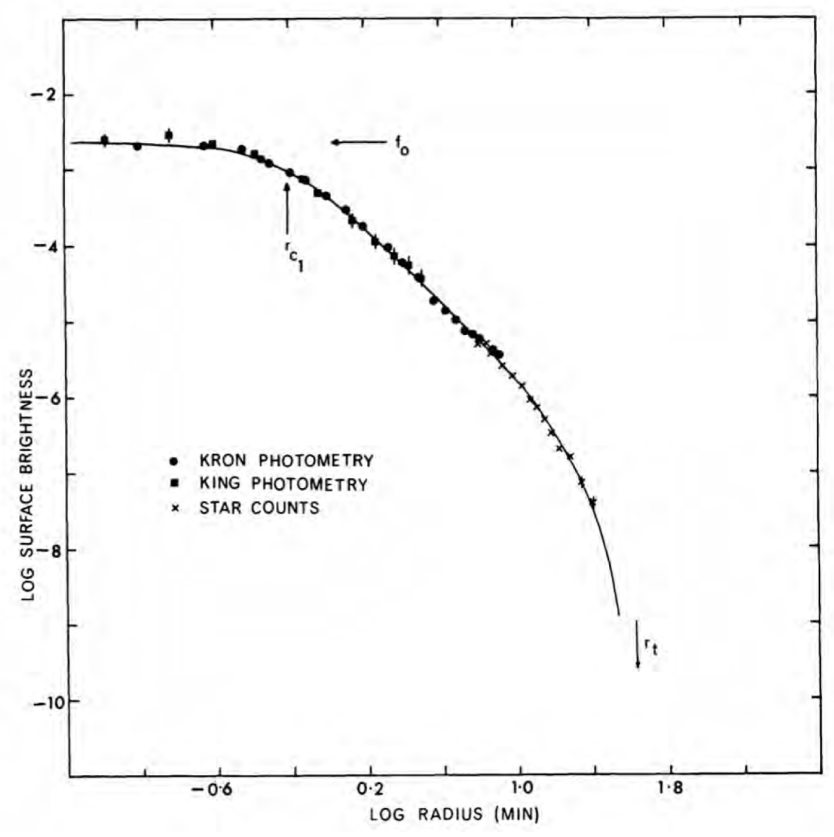

Figure 1: Comparison between the observed surface brightness of the globular cluster M3, given by dots, with the theoretical curve which represent the model developed by Da Costa \& Freeman [ [ $]$ ].

In order to correctly evaluate the influence of mass spectrum in the cluster dynamics, let us consider the number of the stars with various mass values that have been formed in the system. For stars with mass in the range between $m$ and $m+d m$, we have $d N \propto \xi(m) d m$, where $\xi(m)$ is the initial mass function. Salpeter [ए]] found a theoretical power law form $\xi(m) \propto m^{-2.35}$ which is a good approximation for stars with masses $M>0.5 M_{\odot}$. For a more general form of $\xi$ valid in a wider range of masses, we use the mass function introduced by Kroupa [ए]]

$$
\begin{cases}\xi(m) \propto m^{-2.3} & \text { for } \quad m \geq 0.5 M_{\odot} \\ \xi(m) \propto m^{-1.3} & \text { for } \quad 0.1 M_{\odot} \leq m \leq 0.5 M_{\odot} .\end{cases}
$$

We consider a range of star masses in the interval $0.1 M_{\odot} \leq m \leq 1.1 M_{\odot}$ and the condition of normalization given by

$$
\int_{m_{0}}^{m_{1}} e^{m \Delta \varphi / k \theta} \xi(m) d m=1,
$$

where $m_{0}=0.1 M_{\odot}$ and $m_{1}=1.1 M_{\odot}$. The term $e^{m \Delta \varphi / k \theta}$ takes into account the mass segregation effects [2] and $\theta$ is the thermodynamical temperature, constant all over the equilibrium configuration [12]. At this step of development of the model we choose to not include the contribution of white dwarfs and neutron stars, limiting the maximum value of the star masses at $m_{1}=1.1 M_{\odot}$.

In order to make a suitable adaptation of the King models to multimass GCs taking into account the Kroupa mass function, we have to calculate the most important quantities involved in the gravitational equilibrium equation like the number density and the mass density, by using the King distribution function with a particular care to the dependence on the mass. For the number density 
$n(m)$ we have

$$
\frac{N}{V} \equiv n(m)=A(m) e^{m \Delta \varphi / k \theta} \int_{0}^{q_{c}}\left[e^{\varepsilon(q) / k \theta}-e^{\varepsilon_{c}(m) / k \theta}\right] d^{3} q,
$$

where $\Delta \varphi=\varphi(R)-\varphi(r)$ and $A(m)$ is the normalizing factor. The kinetic energy of a single star is $\varepsilon(q)$, while $\varepsilon_{c}(m)$ is the cutoff energy connected with the gravitational potential and depending on the mass of the single star. Integrating on the entire spectrum of masses we obtain the quantity $n(r)$ depending on the radial coordinate

$$
n(r)=\frac{\int_{m_{0}}^{m_{1}} n(m) e^{m \Delta \varphi / k \theta} \xi(m) d m}{\int_{m_{0}}^{m_{1}} e^{m \Delta \varphi / k \theta} \xi(m) d m} .
$$

Analogously, for the mass density $\rho(r)$

$$
\rho(r)=\frac{\int_{m_{0}}^{m_{1}} m n(m) e^{m \Delta \varphi / k \theta} \xi(m) d m}{\int_{m_{0}}^{m_{1}} e^{m \Delta \varphi / k \theta} \xi(m) d m},
$$

where, being in Newtonian regime, $\rho(m)=m n(m)$.

\section{Definition of the main parameters and results}

We calculate the equilibrium configurations of multimass King models by the Poisson equation adapted to the presence of a continuous mass function. The definition of parameters, quite similar to one used by King [ [8]], is introduced taking into account quantities calculated with respect to the maximum mass $m_{1}$ allowed in the model. In particular, the dimensionless cutoff parameter $W$ is given by the formula

$$
W=\frac{\varepsilon_{c, 1}}{k \theta}=\frac{1}{\sigma_{1}^{2}}\left(\varphi_{R}-\varphi\right),
$$

where $\sigma_{1}^{2}=k \theta / m_{1}$ is the square of the velocity dispersion of the maximum mass. In the same way we define the King radius $r_{K, 1}$ with respect to the maximum mass as

$$
r_{K, 1}=\sqrt{\frac{9 \sigma_{1}^{2}}{4 \pi G \rho_{0}}} .
$$

The definition (4. (d) leads to the relation

$$
\frac{d \varphi}{d r}=-\sigma_{1}^{2} \frac{d W}{d r}
$$

and to the final form of the gravitational equilibrium equation

$$
\frac{1}{r^{2}} \frac{d}{d r}\left(r^{2} \frac{d W}{d r}\right)=-\frac{4 \pi G \rho}{\sigma_{1}^{2}} .
$$

Also the radial coordinate $r$ can be adimensionalized with respect to the King radius $r_{K, 1}$ introducing the quantity $\xi=r / r_{K, 1}$, so that the dimensionless form of the gravitational equilibrium equation becomes

$$
\frac{1}{\xi^{2}} \frac{d}{d \xi}\left(\xi^{2} \frac{d W}{d \xi}\right)=-9 \frac{\rho}{\rho_{0}}
$$


with the initial conditions $W(0)=W_{0}$ and $W^{\prime}(0)=0$.

We obtain a family of multimass equilibrium configurations for different values of $W_{0}$. We can compare and contrast these configurations with the single mass models obtained by King [ [8]. Each configuration is characterized by the dimensionless total mass $\mu=M / \rho_{0} r_{K, 1}^{3}$ and the concentration $c=r_{t} / r_{K, 1}$, where the tidal radius $r_{t} \equiv R$ is the value corresponding to the edge of the configuration where $\rho=0$. The dimensionless total mass is simply obtained by using Eq.(4.5) and the definition of $\xi$. We have

$$
\mu=\frac{M}{\rho_{0} r_{K, 1}^{3}}=\frac{1}{\rho_{0} r_{K, 1}^{3}} \int_{0}^{r_{t}} 4 \pi \rho r^{2} d r=4 \pi \int_{0}^{c} \frac{\rho}{\rho_{0}} \xi^{2} d \xi=-\frac{4 \pi}{9}\left(\xi^{2} \frac{d W}{d \xi}\right)_{\xi=c} .
$$

In Fig. $\square$ the behavior of the concentration $c$ and the dimensionless total mass $\mu$ is shown for multimass models and King single mass models at different values of $W_{0}$. We note at same values of $W_{0}$ that multimass models are more extended and massive than King ones. The reason arises from the mass segregation in multimass models where lighter and faster stars occupy the external regions of the cluster making it more extended, while the heavier ones remain in the central regions.
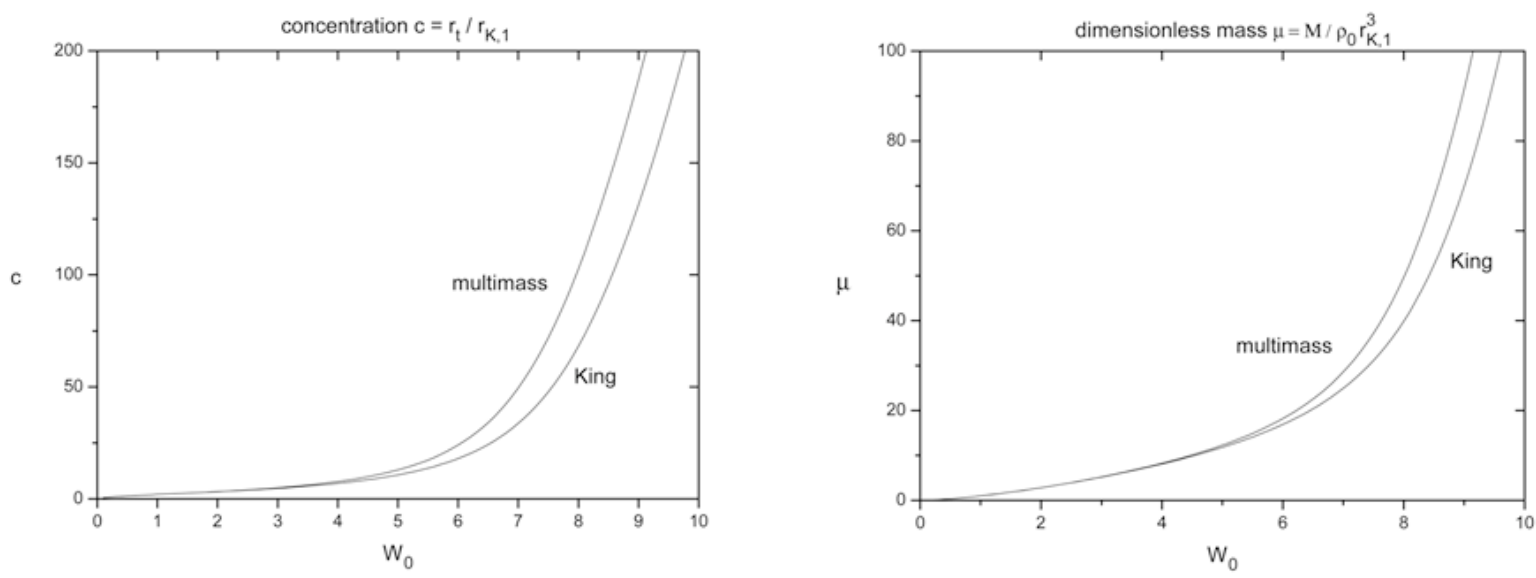

Figure 2: Concentration and dimensionless total mass of multimass equilibrium configurations are compared and contrasted with ones obtained by King [8].

We can also analyze the projected mass density of multimass models at different values of $W_{0}$ and compare the results with ones corresponding to single mass King models. In Fig.[] the plotted quantity $\sigma / \sigma_{0}$ refers to values of the projected mass density with respect to its central value. The expression of $\sigma(r)$, not to be confused with the velocity dispersion $\sigma_{1}$, is given by

$$
\sigma(r)=2 \int_{r}^{r_{t}} \frac{\rho(s) s d s}{\sqrt{s^{2}-r^{2}}} .
$$

This quantity is the relevant one in the comparison with the surface brightness of the observed clusters, in the hypothesis that the mass-luminosity ratio $M / L$ remains quite constant in the observed system. It is interesting to note that models with the same concentration $c$ (both multimass and King ones) show pratically the same profile. This could be the reason which allows a good fit between the observed surface brightness of the clusters with single mass King models, in spite of the presence of stars with different masses in GCs. 

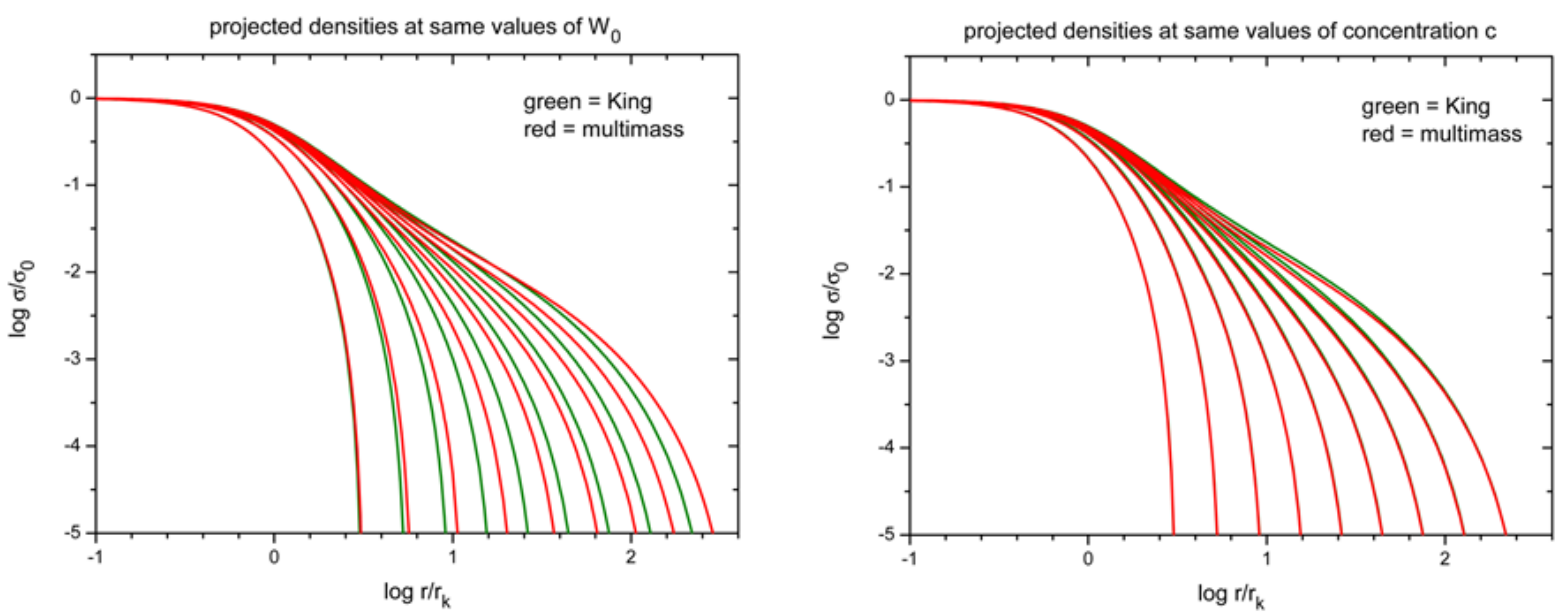

Figure 3: Projected mass density of multimass and King models with respect to radial coordinate at same values of the parameter $W_{0}$ and the concentration $c$, respectively.
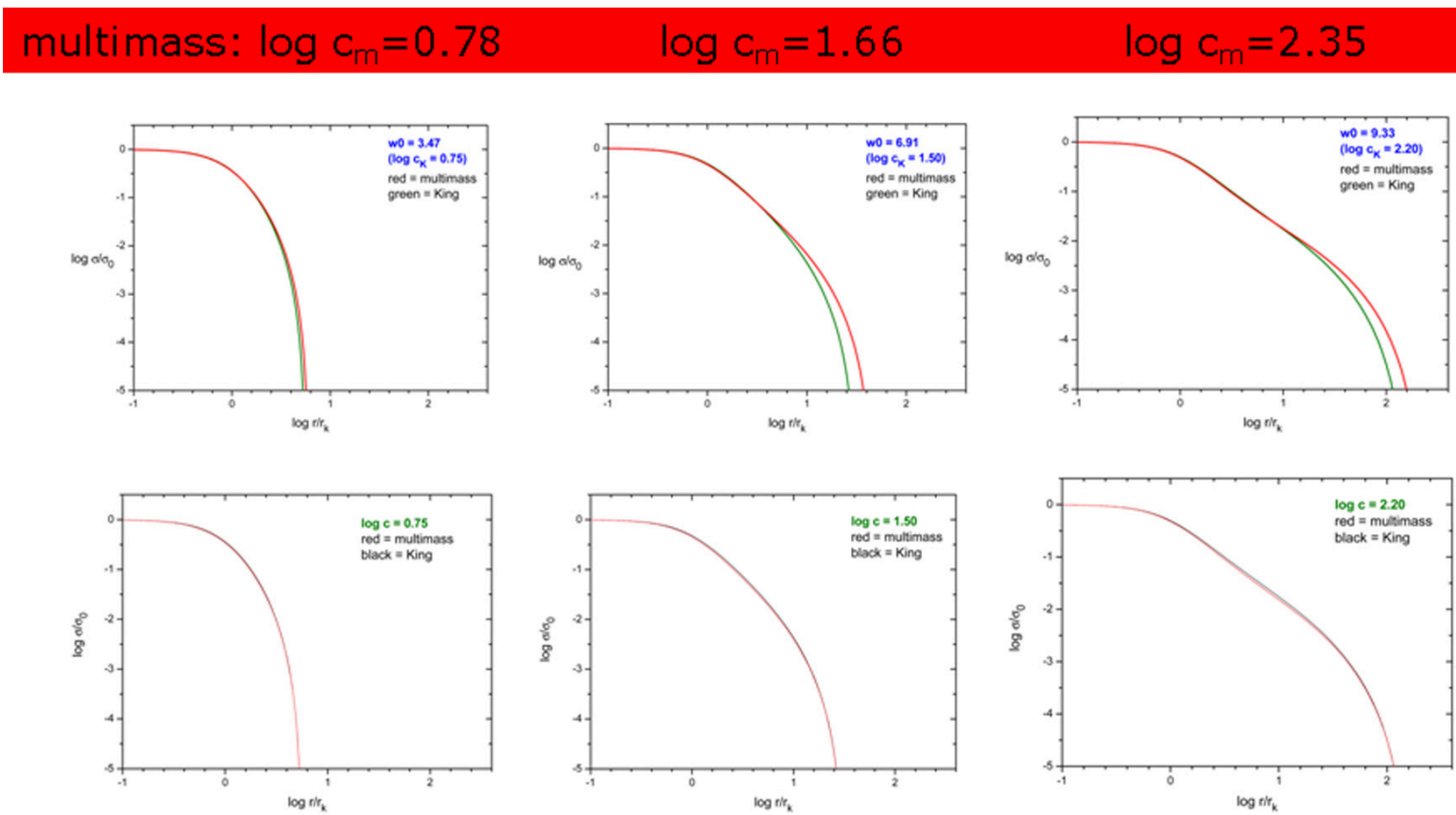

$$
\text { multimass: } W_{0 m}=3.30 \quad W_{0 m}=6.40 \quad W_{0 m}=8.70
$$

Figure 4: Projected mass densities for three fixed values of the parameter $W_{0}$ and the concentration $c$ for both models. 
Even more interesting is the analysis shown in Fig.t. In the first three plots (top of figure) we can see the behavior of normalized projected mass density $\sigma / \sigma_{0}$ for three fixed values of the parameter $W_{0}$. In each plot the profile of the multimass model is compared with the King one. These profiles are different, especially in the external regions, and, even if they have the same value of $W_{0}$, they correspond to different values of the concentration $c$. In the last three plots (bottom of figure) we can see the behavior of the same quantity for three fixed values of the concentration $c$. In each plot the profiles of both models overlap in spite of the different value of $W_{0}$. The conclusion is that the concentration is the relevant quantity which characterizes the profiles and it seems unaffected by the choice of the model: multimass models and King models with equal concentration have exactly the same profile.
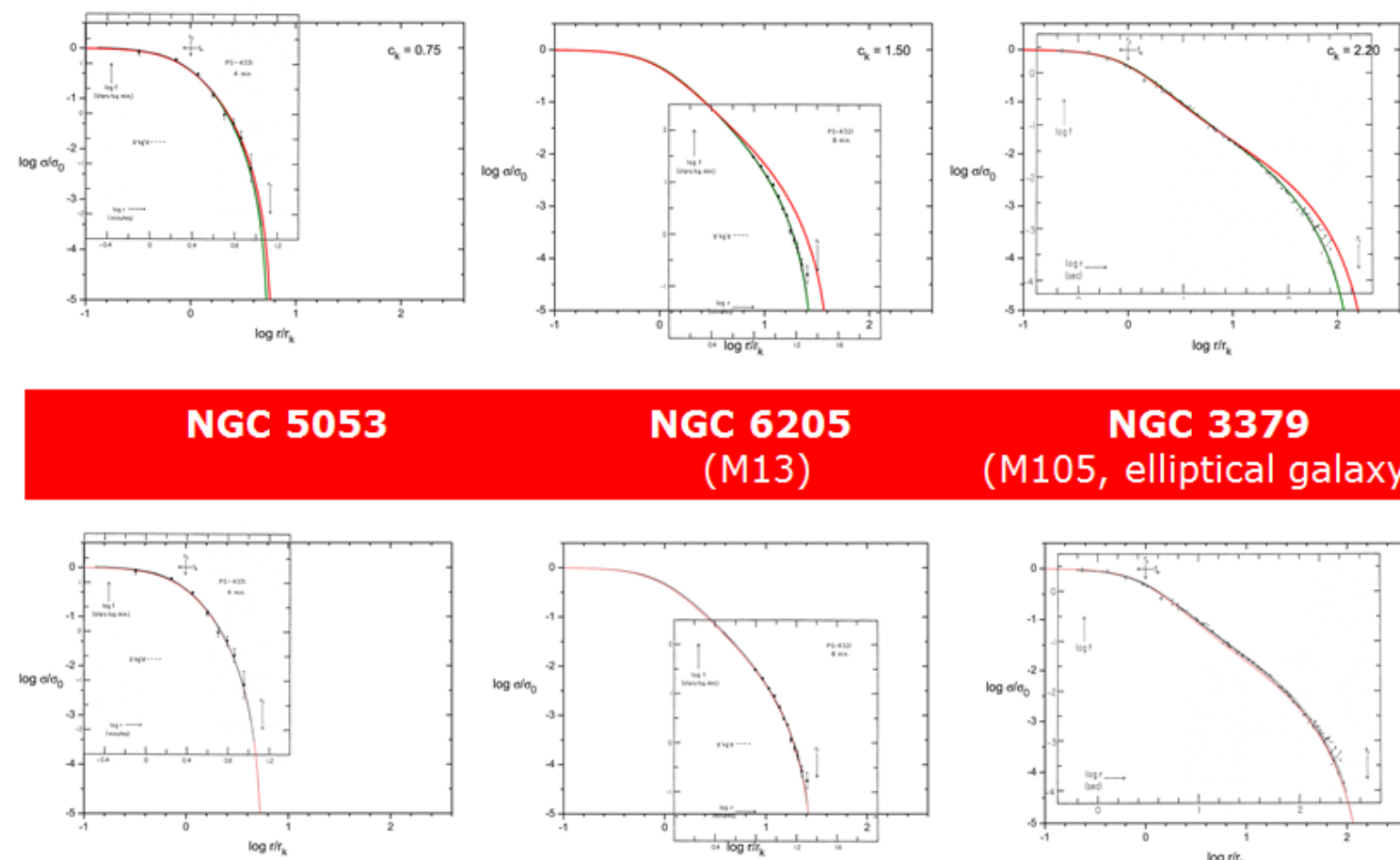

NGC 6205 (M13)

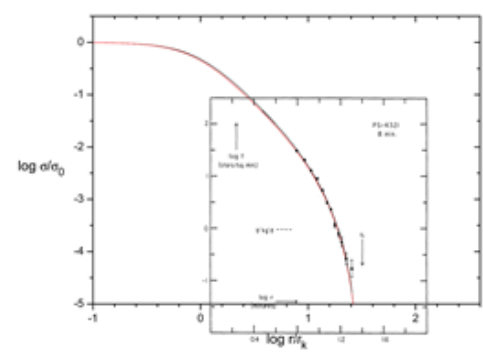

NGC 3379 (M105, elliptical galaxy)

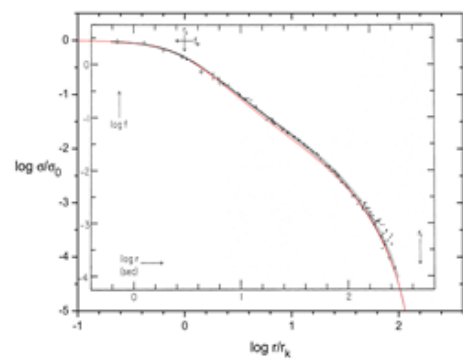

Figure 5: Observational data from three clusters: NGC 5053 and NGC 6205 (globular clusters), and NGC 3379 (elliptical galaxy). Theoretical projected mass densities of both models (multimass and King) fit data for specific values of the concentration. Fits with King models are due to King [8]].

Finally, in Fig.[1, observational data from three different clusters (NGC 5053, NGC 6205 and NGC 3379) are compared with the profiles of both multimass and King models. The choice of the fitting values of the concentration is given by King [ [ $]$ ]. Note that also the multimass model with the same value of the concentration fits the observational data, leading to the conclusion that also the multimass model is able to fit every surface brightness of GCs like the King one.

\section{Mass segregation and cluster instability}

Mass segregation is an important effect we can observe in GCs, separating heavier masses from lighter ones in different regions. This phenomenon may be observed also in numerical simulations 
with multimass population of stars. In our multimass theoretical model the segregation effect is also clearly present as shown in Fig.6, due to the presence of Spitzer term $e^{m \Delta \varphi / k \theta}$. We can see that heavier masses are prevailing at larger values of $W$, corresponding to central regions of the clusters. At $W=0$ we recover the pure Kroupa mass function defined by Eq.(B.].

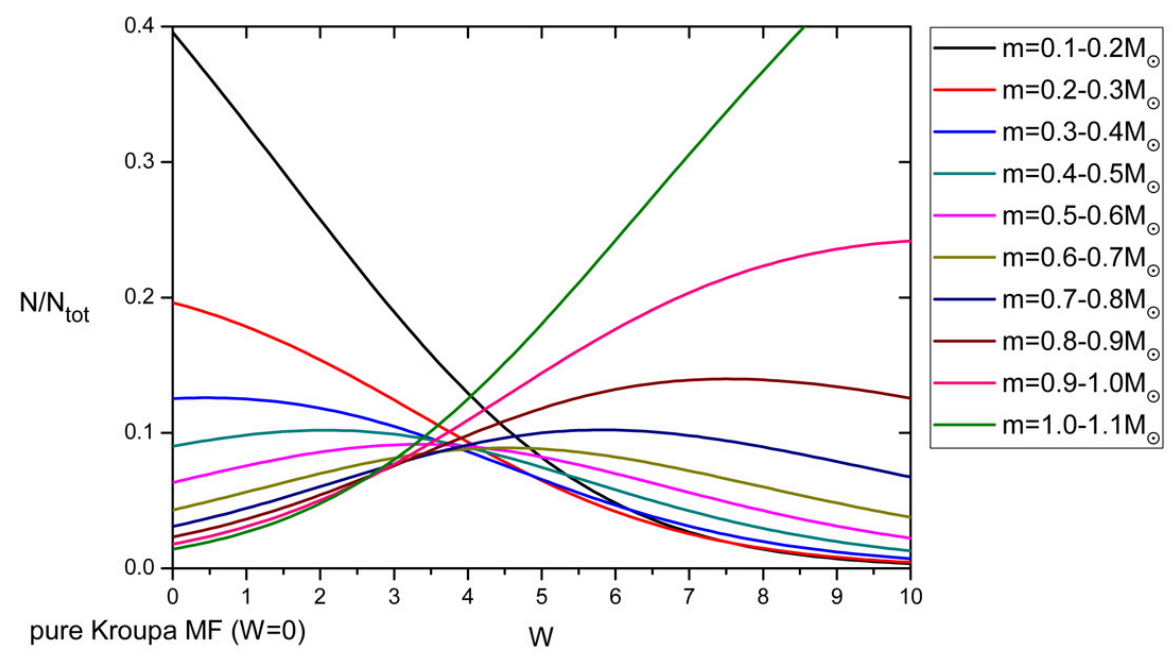

Figure 6: Effect of mass segregation on Kroupa mass function. Larger values of $W$ correspond to central regions where the gravitational potential is increasing in absolute value.

Mass segregation is an important factor in the evolution of GCs and the thermodynamical stability of the system is greatly affected by this phenomenon. Mass segregation induces an increasing of the star evaporation and tidal forces prevail on gravity if the mass distribution is not well tuned: the most part of lighter and faster stars leave the system and are captured by the gravitational potential of the hosting galaxy. This effect also influences the process of virialization and equipartion of the clusters making the system unstable with respect to tidal disgregation. This connection among these different effects is not yet well understood and remains one of the open problems of stellar dynamics of GCs (see e.g. [ए]][[20][[ए8]) which could be called the ESEV puzzle (Equipartition $\rightarrow$ Segregation $\rightarrow$ Evaporation $\rightarrow$ Virialization).

This instability is clearly visible in Fig. $\square$ where the total energy including the contribution of the effective potential [ए2] is plotted in function of $W_{0}$. Here the total energy, due to the virial condition, can be rewritten as $E_{t o t}=-E_{k i n}+E_{\text {eff }}[[\mathbb{[} 3]$. Differently from the single mass King model, where the mass segregation is not present, the total energy for the multimass model is always in the positive values region, leading to the conclusion that the system is unstable with respect to disgregation due to tidal forces induced by the hosting galaxy. Also the other multimass models existing in literature suffer the same problem.

The instability for tidal disgregation is also present in King models but only for $W_{0} \leq 3$ [[13]. In this case, due to small gravitational potential not sufficient to contrast the tidal forces of the hosting galaxy, the evaporation of stars leads to the deplition of the stellar population, making the system not able to maintain bound itself. This is the case of Koposov systems, well known in literature [ए]], which have values of $W_{0}$ lesser than 3 and therefore they are fated to tidal disruption. 


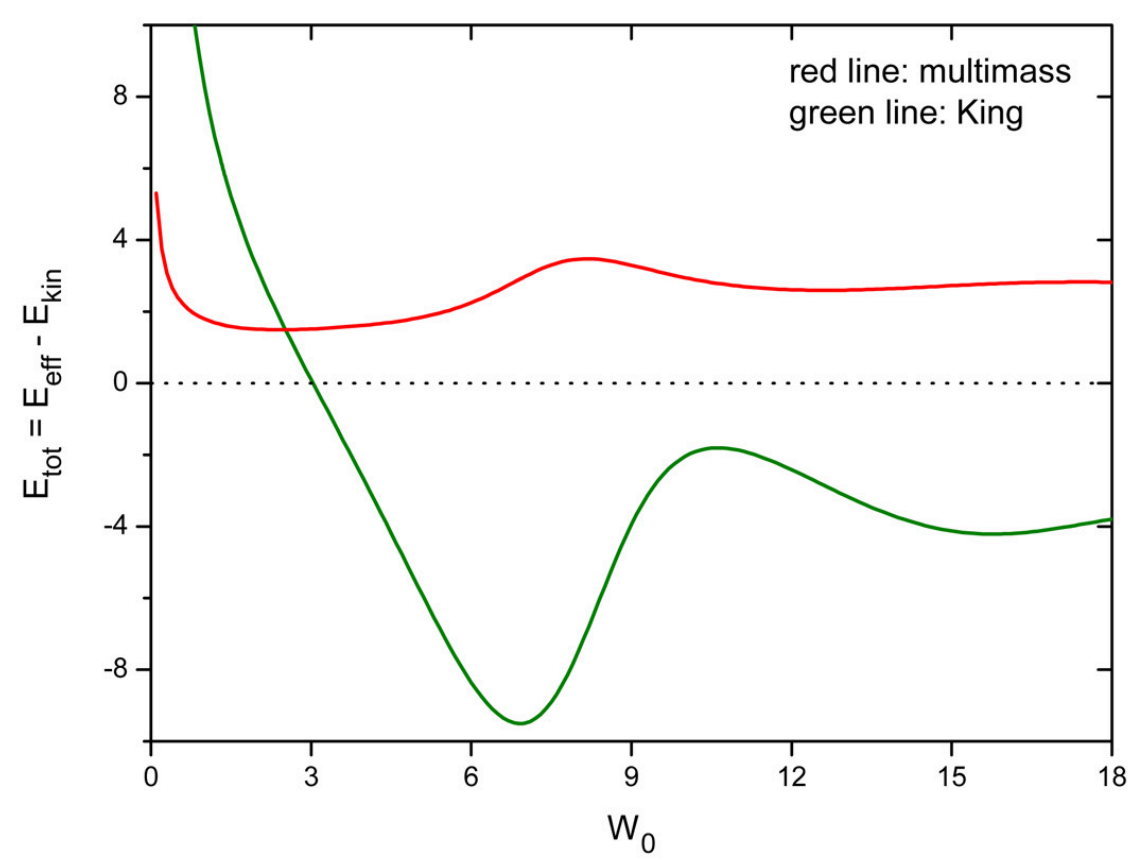

Figure 7: Total energy of the system, including the effective potential contribution at different values of $W_{0}$ for multimass and King models.

\section{Conclusions}

At equal concentration, the value of $W_{0}$ of each model obtained by considering a multimass function sistematically decrease with respect to the corresponding King model, shifting the GCs distribution in $W_{0}$ at lesser values. Differences in projected density with the corresponding King model at equal value of $W_{0}$ are appreciable only in the external region at $r>r_{k}$. Differently, models with the same concentration $c$ show identical profiles both in multimass and single mass (King) case.

Moreover, while in the King models the possibility to obtain positive values of the total energy of the clusters, due to presence of the effective potential, gives the opportunity to explain the conditions of disruption of the clusters at very small values of $W_{0}$ where the tidal forces prevail on the gravitational ones, and these conditions are in accordance with the observations and several $\mathrm{N}$-body simulations, in multimass models the mass segregation makes unstable each configuration, being the total enengy always positive. This is an open problem.

Measurements of transversal star velocities allowing to analyze the real kinematics in GCs showed problems in order to verify the possible phenomenon of the equipartition in presence of mass segregation [1[7]. Nevertheless such measurements can give the possibility to test multimass model and check the existence of the effective potential from observational data, instead of using numerical simulations.

Similarly, observations on kinematics of stars can be useful for giving information about mass segregation, in order to set in the better way the factor $A(m)$ and solve the problem of the total 
energy. This is important for a correct analysis of the thermodinamical instability of the system, through the calculation of the effective potential related to tidal forces induced by the hosting galaxy. In this way also the ESEV puzzle could have a decisive clarification.

Definitely, the model must be expanded for including the presence of neutron stars and white dwarfs and (possibly) taking into account the different contribution in luminosity of stars in the calculation of the projected mass density.

Finally, it is necessary to improve the observations to detect fainter objects and avoid possible observative selection effects, in order to obtain data from M31 and NGC5128 useful to implement a unique $W_{0}$-distribution valid for different GCs-galaxy systems.

\section{References}

[1] Binney, J., Tremaine, S. 1987, Galactic Dynamics, Princeton Univ. Press, Princeton

[2] Da Costa, G.S., Freeman, K.C. 1976, AJ, 206, 128

[3] Da Costa, G.S. 1982, AJ, 87, 990

[4] Gieles, M., Zocchi, A. 2015, MNRAS, 454, 576

[5] Gunn, J.E., Griffin, R.F. 1979, AJ, 84, 752

[6] Harris, W.E. 1996, AJ, 112, 1487

[7] Harris, W.E. 2013, arXiv:1306.2241v1

[8] King, I.R. 1966, AJ, 71, 64

[9] Koch, A., Grebel, E.K., Odenkirchen, M., Martinez-Delgado, D., Caldwell, J.A.R. 2004, AJ, 128, 2274

[10] Koposov, S., de Jong, J.T.A., Belokurov, V., Rix, H.W., Zucker, D.B., Evans, N.W., Gilmore, G., Irwin, M.J., Bell, E.F. 2007, ApJ, 669, 337

[11] Kroupa, P. 2001, MNRAS, 322, 231

[12] Merafina, M., Vitantoni, D. 2014, Acta Polytechnica CTU Proc., 1, 231

[13] Merafina, M. 2016, Mem. S.A.It., 87, 583

[14] Merafina, M. 2018, PoS (MULTIF2017), 004

[15] Michie, R.W. 1963, MNRAS, 125, 127

[16] Miocchi, P. 2006, MNRAS, 366, 227

[17] Parker, R.J., Goodwin, S.P., Wright, N.J., Meyer, M.R., Quanz, S.P. 2016, MNRAS, 459, L119

[18] Peuten, M., Zocchi, A., Gieles, M., Hénault-Brunet, V. 2017, 470, 2736

[19] Salpeter, E.E. 1955, ApJ, 121, 161

[20] Sollima, A., Dalessandro, E., Beccari, G., Pallanca, C. 2017, MNRAS, 464, 3871

[21] Spitzer, L. 1987, Dynamical Evolution of Globular Clusters, Princeton Univ. Press, Princeton

[22] Zhang, C., Li, C., de Grijs, R., Bekki, K., Deng, L., Zaggia, S., Rubele, S., Piatti, A.E., Cioni, M.L., Emerson, J., For, B., Ripepi, V., Marconi, M., Ivanov, V.D., Chen, L. 2015, ApJ, 815, 95 


\section{DISCUSSION}

JORDI ISERN QUESTION: How white dwarf distribution is affected by this formulation?

MARCO MERAFINA ANSWER: At this step of development of the model I did not include the presence of white dwarfs and neutron stars. As I wrote in the conclusions, I plan to improve the model with the presence of such kind of stars. The presence of these stars mainly affects the mass segregation with an increase of the concentration of heavier stars in the innermost region of the cluster. This also affects the evaporation of stars which is greatly increased, making the system more unstable. Another effect is the increase in binary systems formation leading to a relevant contrast to the energy equipartion process.

DMITRY BISICALO QUESTION: How the dissipation processes change the solution?

MARCO MERAFINA ANSWER: The solution is given by the Fokker-Planck equation which takes into account the orbit diffusion of stars. Collisions are elastic in almost all the cases. We have only an exchange of kinetic energy which tends to diffuse in phase space, but this phenomenon is contrasted by the star evaporation which keeps constant the form of the distribution function. Evolution is therefore characterized by the changement of the parameters, like in a thermodynamic transformation. 